\title{
Conchas de moluscos com vestígios de utilização por seres humanos encontradas no Sambaqui do Boguaçu, Guaratuba, Paraná
}

\author{
Marcos de Vasconcellos Gernet ${ }^{1}$ (D), Esther Valentina da Veiga de Vasconcellos \\ Gernet $^{1}$ (D) \& Elizângela da Veiga Santos ${ }^{1,2}$ (D)
}

(1) Universidade Federal do Paraná, Laboratório de Ecologia Aplicada e Bioinvasões, Rua Rio Grande do Norte 145, Mirassol 83255-000, Pontal do Paraná, Paraná, Brasil. E-mail: lmv.gernet@gmail.com

(2) Universidade Federal do Paraná, Setor Litoral, Rua Jaguariaíva 512, Caiobá 83260-000, Matinhos, Paraná, Brasil. E-mail: lizveiga.ga@gmail.com

Gernet M.V., Gernet E.V.V.V. \& Santos E.V. (2019) Conchas de moluscos com vestígios de utilização por seres humanos, encontradas no Sambaqui do Boguaçu, Guaratuba, Paraná. Pesquisa e Ensino em Ciências Exatas e da Natureza, 3(2): 142-146. http://dx.doi.org/10.29215/pecen.v3i2.1260

Editor acadêmico: Silvio F. B. Lima. Recebido: 14 Junho 2019. Aceito: 11 Setembro 2019. Publicado: 02 Outubro 2019.

Resumo: Os sambaquis são definidos como sítios arqueológicos, localizados na paisagem holocênica do Período Quaternário. A fauna malacológica é o principal constituinte dos sambaquis, influenciando na sua composição química e estrutural. $\mathrm{O}$ sambaqui do Boguaçu, com $7 \mathrm{~m}$ de altura, localiza-se nas margens do rio de mesmo nome, o qual desemboca na baía de Guaratuba. Ao todo, foram encontrados e analisados 10 exemplares de cinco espécies distintas de moluscos marinhos pertencentes a cinco famílias, que apresentavam vestígios de utilização por seres humanos.

Palavras chave: Sítio arqueológico, raspadores, indícios.

Shells of mollusks with traces of use by humans found in Sambaqui do Boguaçu, Guaratuba, Paraná

Abstract: The sambaquis are defined as archaeological sites, located in the Holocene landscape of the Quaternary Period. Malacological fauna is the main constituent of sambaquis, influencing its chemical and structural composition. The Boguaçu sambaqui has $7 \mathrm{~m}$ high and is located on the banks of the river of the same name, which ends at Guaratuba Bay. In all, 10 specimens of five distinct species of marine mollusks belonging to five families were found and analyzed, which showed traces of use by humans.

Key words: Archaeological site, scraper, clues.

O termo sambaqui deriva do Tupi e significa ( Tamba = conchas e $K \boldsymbol{K}=$ amontoados) (Gaspar et al. 2011). Os sambaquis são definidos como sítios arqueológicos, localizados na paisagem holocênica (planície litorânea) do Período Quaternário (Gernet \& Birckolz 2011). Tais monumentos são formações artificiais (construídas pelo homem), caracterizadas pela presença de grande quantidade de conchas de moluscos e, em menor número, de restos de peixes e outros animais, associados a instrumentos líticos e ósseos, esqueletos humanos, estruturas usadas para habitação e restos de fogueiras, formando colinas que podem alcançar mais de $30 \mathrm{~m}$ de altura (Prous 1992). Sambaquis foram construídos por antigos grupos sociais conhecidos como pescadores-caçadores-coletores servindo basicamente como área de acampamento, estando presentes em praticamente todo o litoral brasileiro, concentrados, principalmente, em regiões estuarinas, lagunares e enseadas (Schmitz 1984; De Masi 2009; Godino et al. 2011).

A fauna malacológica é o principal constituinte dos sambaquis, influenciando na sua composição química e estrutural. Através dela podemos inferir sobre o ambiente pretérito em que foram construídos estes monumentos. O conhecimento a respeito dos moluscos é 
importante para se efetuar uma reconstrução paleoambiental dos sambaquis comparando a abundância das espécies deste filo no passado e nos dias atuais (Prous 1990). No Brasil, as populações sambaquieiras colonizaram intensamente toda a costa, principalmente em ambientes lagunares (Scheel-Ybert 2001; Guimarães 2003). Assim, existem cinco regiões lagunares onde a presença de sambaquis é bastante acentuada: Cananéia-Iguape em São Paulo, baía de Paranaguá no Paraná, a região de São Francisco do Sul e Laguna em Santa Catarina (Okumura \& Eggers 2005). Segundo o trabalho de Neves et al. (2005), que analisaram aspectos culturais em sambaquis brasileiros, correlacionando utensílios confeccionados em conchas e rochas com os hábitos alimentares, as comunidades sambaquieiras do sul do país eram inter-relacionadas por sítios que apresentavam objetivos funcionais diferenciados, perceptivos em diferentes épocas de sua construção pela independência funcional entre suas camadas.

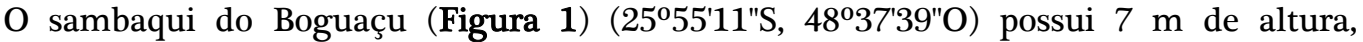
localiza-se nas margens do rio de mesmo nome e desemboca na baía de Guaratuba (Gernet \& Birckolz 2011). Cronologicamente, situa-se entre 4000 e 1500 anos atrás. Durante muitos anos, assim como outros sambaquis do litoral paranaense, foi explorado e suas conchas utilizadas para fabricação de cal e pavimentação de estradas. Atualmente, encontra-se ameaçado pela ação das marés e também pelo vandalismo.

Durante dois anos, o primeiro autor participou com um grupo de pesquisadores de um projeto de avaliação de bactérias do solo no entorno do sambaqui do Boguaçu. Durante a execução do projeto, ao caminhar no entorno do sambaqui, o primeiro autor notou a presença de conchas de moluscos provenientes deste sítio, e que apresentavam alterações diretamente relacionadas ao uso por seres humanos. A falta de licença por parte do Instituto do Patrimônio Histórico e Artístico Nacional (IPHAN) e da permissão de coleta de material possibilitou apenas a prospecção visual direta e realização de fotografias das conchas em campo. Sendo assim, as conchas de moluscos encontradas, que apresentavam indícios de manuseio pelo homem do sambaqui, não foram coletadas, apenas analisadas e fotografadas em campo utilizando-se de máquina fotográfica digital e escala de $1 \mathrm{~cm}$.

A identificação taxonômica foi feita através de literatura especializada (Rios 2009; Souza et al. 2011) e atualizada com base no WORMS Editorial Board (2019). Os diferentes instrumentos e adornos encontrados, confeccionados em conchas, foram identificados e classificados levandose em consideração a morfologia e modo de manufatura (Bennyhoff \& Hughes 1987).

Ao todo foram encontrados e analisados 10 exemplares de cinco espécies distintas de moluscos marinhos pertencentes a cinco diferentes famílias. As conchas encontradas apresentavam as seguintes funcionalidades: três exemplares de Cyrtopleura costata (Linnaeus, 1758) (Figura 2A) e dois exemplares de Olivancillaria urceus (Gmelin, 1791) (Figura 2B), apresentavam perfurações com polimento para serem utilizadas como adorno (provavelmente peitoral); um exemplar de Tivela zonaria (Lamarck, 1818) (Figura 2G) e um exemplar de Phacoides pectinatus (Gmelin, 1791) (Figura 2D) com serrilhados contínuos na borda, funcionando como faca ou raspador, o que pode ser observado apenas em bivalves; três exemplares de lábio externo de Semicassis granulata (Born, 1778) (Figura 2E) cujas extremidades encontravam-se polidas, funcionando como anzol ou perfurador.

As conchas que estavam perfuradas (Figuras 2A-B) apresentavam sinais profundos de polimento, com estrias paralelas e compridas evidenciando ação humana e não uma marca natural de predação, como também verificado no trabalho de Klokler (2014) para as chamadas contas discóides. No caso do bivalve Cyrtopleura costata, a perfuração encontrava-se no umbo, o que é comum em artefatos manufaturados, como também observados nos trabalhos de Prous (1990) e Kokler (2014). Nas espécies de bivalves com serrilhados na borda (Figuras 2C-D), percebe-se nitidamente trabalhos de picoteamento produzidos possivelmente por instrumentos líticos, dando o aspecto serrilhado como observado no trabalho de Aizpurua \& Mcanany (1999) cuja função principal é de raspador, sendo utilizado na retirada de pele de pequenos animais e também para raspar a casca de plantas. 


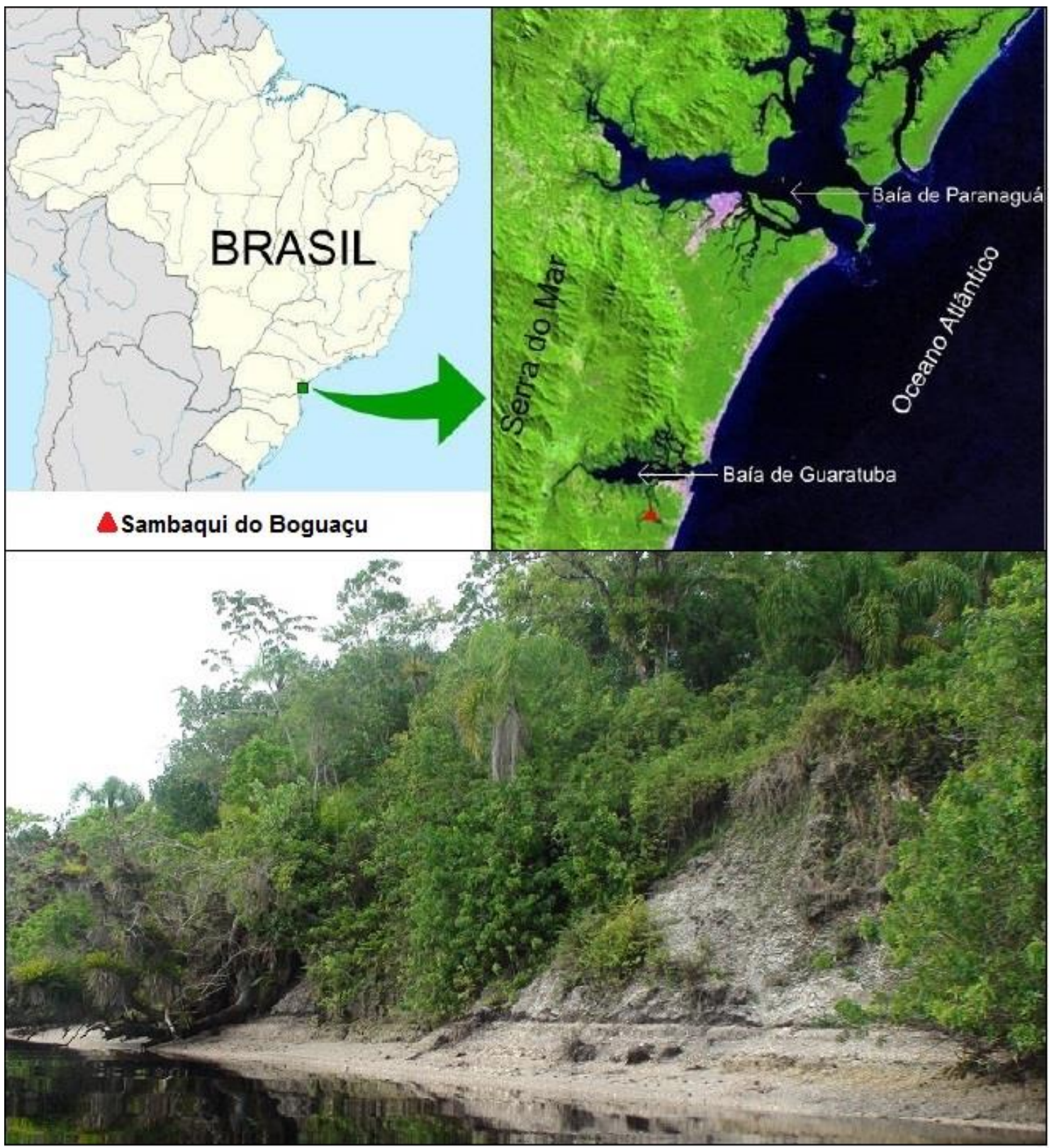

Figura 1. Mapa e foto do Sambaqui do Boguaçu, baía de Guaratuba, Paraná.

Neste trabalho foi possível verificar a ocorrência de três novas espécies [Olivancillaria urceus (Figura 2B), Tivela zonaria (Figura 2C) e Semicassis granulata (Figura 2E)] para o sambaqui do Boguaçu, não registradas no trabalho de Gernet \& Birckolz (2011).

Para Klokler (2014), a seleção de conchas para a manufatura de adornos é creditada à grande variedade de formas, cores e atributos dessas matérias-primas, o que as torna tecnologicamente versáteis. Todas as espécies identificadas são encontradas ainda hoje na área de influência do sambaqui.

As conchas, além de esclarecerem detalhes sobre os hábitos alimentares dos povos construtores de sambaquis, também fornecem dados a respeito dos instrumentais utilizados por estas populações pré-históricas. Portanto, não é só quantitativamente que os moluscos são importantes dentro de um sambaqui, mas também qualitativamente. É perceptível que a dureza da concha e suas características estruturais, definem o tamanho do objeto a ser produzido e sua funcionalidade. 


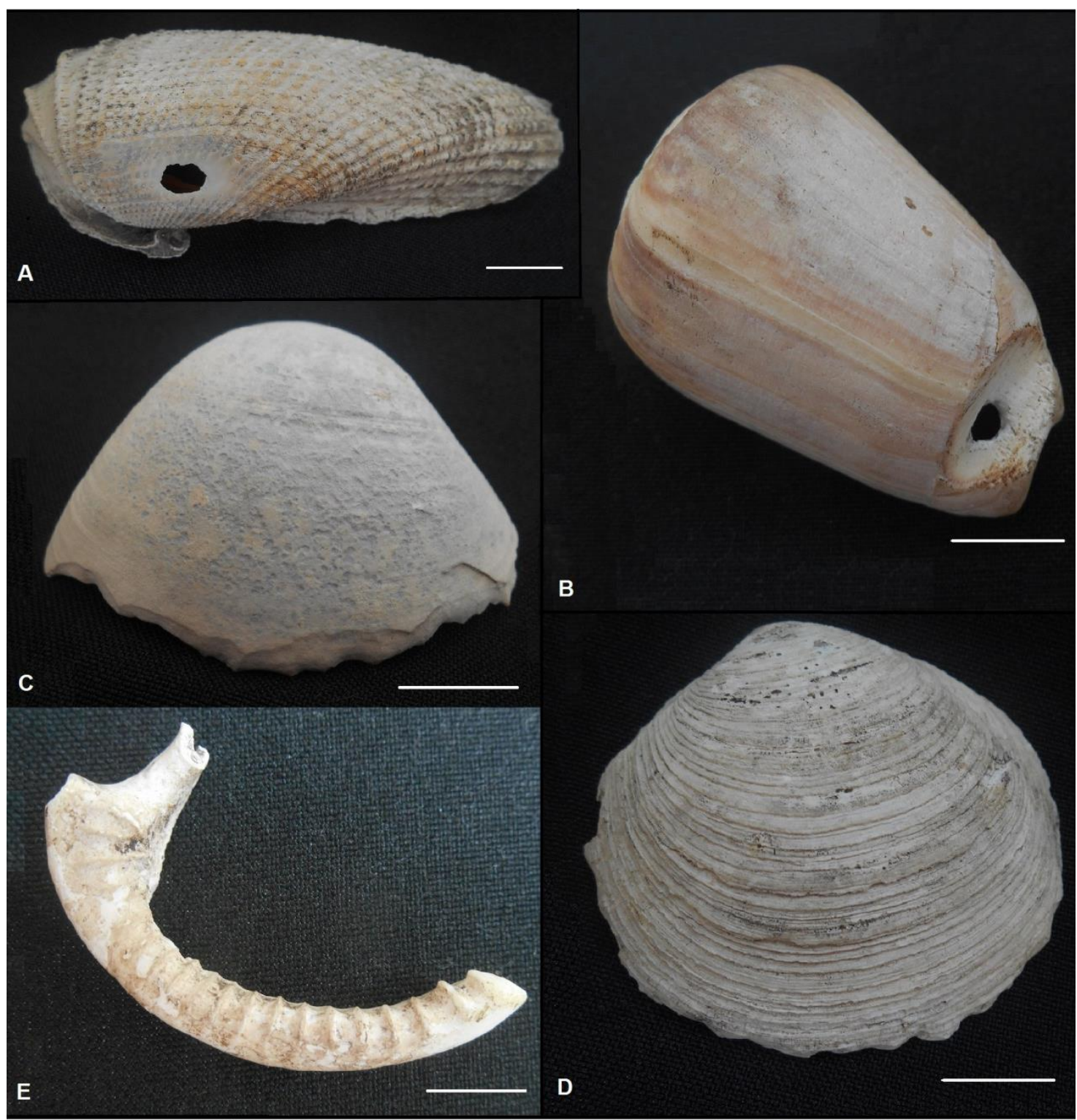

Figura 2. Conchas com vestígios de utilização por seres humanos encontradas no sambaqui do Boguaçu (barra de escala: $1 \mathrm{~cm}$ ): A. Cyrtopleura costata, B. Olivancillaria urceus, G. Tivela zonaria, D. Phacoides pectinatus, E. Semicassis granulata.

\section{Agradecimentos}

Aos revisores pelas considerações que contribuíram para enriquecer o manuscrito.

\section{Referências}

Aizpurua I.I.I. \& Mcanany P.A. (1999) Adornment and Identity: Shell ornaments from Formative K'axob. Ancient Mesoamerica, 10: 117-127. DOI: 10.1017/S095653619910107X

Bennyhoff J.S. \& Hughes R. (1987) Shell Bead and Ornament Exchange Networks between California and the Western Great Basin. Anthropological Papers of the American Museum of Natural History, 64: 79-175.

De Masi M.A.N. (2009) Aplicações de isótopos estáveis de 18/16 O, 13/12 C e 15/14 N em estudos de sazonalidade, mobilidade e dieta de populações pré-históricas no sul do Brasil. Revista de Arqueologia, 22: 55-76. 
Gaspar M.D., Klokler D. \& De Blasis P. (2011) Traditional fishing, mollusk gathering, and the shell mound builders of Santa Catarina, Brazil. Journal of Ethnobiology, 31: 188-212. DOI: 10.2993/0278-0771-31.2.188

Gernet M.V. \& Birckolz C.J. (2011) Fauna malacológica em dois sambaquis do litoral do Estado do Paraná, Brasil. Santa Catarina. Biotemas, 24: 39-49. DOI: 10.5007/2175-7925.2011v24n3p39

Godino I.B., Álvarez M., Balbo A., Zurro D., Madella M., Villagrán X. \& French C. (2011) Towards high-resolution shell midden archaeology: Experimental and ethnoarchaeology in Tierra del Fuego (Argentina). Quaternary International, 239: 125-134. DOI: 10.1016/j.quaint.2011.04.017

Guimarães M.S.B.C. (2003) Do lixo ao luxo: As premissas teórico-metodológicas e a noção de sambaqui. Rio de Janeiro. Boletim do Museu Nacional, 63: 1-23.

Klokler D. (2014) Adornos em concha do sítio Cabeçuda: Revisita às amostras de Castro Faria. Revista de Arqueologia, 27: 150-169. DOI: 10.24885/sab.v27i2.408

Neves W.A., Hubbe M., Okumura M.M.M., González-José R., Figuti L., Eggers S. \& De Blasisc P.A.D. (2005) New early Holocene human skeleton from Brazil: implications for the settlement of the New World. Journal of Human Evolution, 48: 403-414. DOI: 10.1016/j.jhevol.2004.12.001

Okumura M.M.M. \& Eggers S. (2005) The people of Jabuticabeira II: reconstruction of the way of life in a Brazillian shellmound. Journal of Comparative Human Biology, 55: 263-281.

Prous A. (1990) Os Moluscos e a Arqueologia Brasileira. Belo Horizonte. Arquivos do Museu de História Natural da UFMG, 11: 241-298.

Prous A. (1992) Arqueologia brasileira. Brasília: Editora UnB-Universidade de Brasília. 609 p.

Rios E.C. (2009) Compendium of Brazilian Sea Shells. Rio Grande: Evangraf. 412 p.

Scheel-Ybert R. (2001) Man and vegetation in the Southeastern Brazil during the Late Holocene. Journal of Archaeological Science, 28: 471-480. DOI: 10.1006/jasc.2000.0577

Schmitz I.A. (1984) Caçadores e coletores da pré-história do Brasil. São Leopoldo: Instituto Anchietano de Pesquisas. $63 \mathrm{p}$.

Souza R.C.L., Lima T.A. \& Silva E. (2011) Conchas marinhas de sambaquis do Brasil. Rio de Janeiro: Technical Books Editora. 256 p.

WORMS Editorial Board (2019) World Register of Marine Species. Disponível em: www.marinespecies.org (Acessado em 14/05/2019). 